Original Research Paper

\title{
Gravity, the Origin of the Mass in the Cosmos
}

\author{
Francisco Pavía Alemany and Marcelino Alvarez Villarroya \\ Agrupación Astronómica de la Safor, Cosmología, Gandía, España, Spain
}

\author{
Article history \\ Received: 29-06-2021 \\ Revised: 11-08-2021 \\ Accepted: 12-08-2021 \\ Corresponding Author: \\ Marcelino Alvarez Villarroya \\ Agrupación Astronómica de la \\ Safor, Cosmología, Gandía, \\ España \\ Email: maralvilla@gmail.com
}

\begin{abstract}
The most accepted theory for the evolution of the Cosmos is the Big Bang theory, which suggests that, at the beginning, the entire mass-energy of the Cosmos was concentrated within an extremely small, dense and hot singularity. Here, we present a new physical formula that, although obtained in an extremely simple way, has significant implications in Cosmology. This equation indicates that the mass of the Cosmos has grown proportionally with time. This growth equates to a Planck mass for each unit of Planck Time, which is, the mass of 200000 suns per second. Finally the total energy of the Cosmos is demonstrated to be zero.
\end{abstract}

Keywords: Cosmology, Astrophysic, Big Bang, Gravity, Zero-Energy Cosmos, Change of Paradigm

\section{Introduction}

At the end of the 19th century, most scientists were convinced that in the field of physics, there was little to be done that all the specialties within the discipline were complete and that, at most, one decimal point could be added to the values of some constants.

It was precisely at the end of the 19th century and the beginning of the 20th century that the greatest progress and transformation took place in this discipline. Those huge advances were made by Max Planck, with its Quantum Theory and by Albert Einstein, with its Theory of Relativity.

At that time, scientists and educated men had an idea of the Universe that was quite generalized and accepted: That of a static and eternal cosmos, that is, without changes and without a beginning or end in time, in addition to being very minute and only constituted by the objects of our own galaxy.

This conception of the cosmos is the one that Albert Einstein defended for a long time. He was so convinced of this hypothesis, that he was forced to introduce "ad hoc" the Cosmological Constant in his General Relativity Equation, to be consistent with the hypothesis of a stable and static universe, despite of the warnings of Fridman (1922), (Lemaître, 1927) and Hubble (1929) and others, who indicated the possibility of an expanding Universe, to whicah he always responded with harsh tones.

When Edwin Hubble demonstrated that the Cosmos is expanding (Hubble, 1929), the hypothesis of the Static Cosmos ceased to make sense. However, Einstein was still convinced that this hypothesis was true. When Einstein visited Hubble in 1931 at the Mount Wilson Observatory, which had the world's most powerful 254-centimeter telescope (Isaacson,
2008) and saw the evidence presented by Edwin, he had to admit that he «had made the greatest mistake of his life» by inserting the Cosmological Constant into his equation of General Relativity (Gamow, 1970).

Several researchers saw the need to replace the incorrect hypothesis with a new one, which, despite of the expansion of the Cosmos, would translate into minimum changes to the original idea. Fred Hoyle and others developed a new theory of a Stationary Cosmos, in which mass is continuously being created to maintain the constant density of the Universe (Hoyle, 1948).

George Gamow proposed another alternative, the Big Bang theory, based on the ideas of the Jesuit priest Lemaitre about the expansion of the universe and referred to as "the hypothesis of the primeval atom" or the "cosmic egg" (Lemaitre, 1927). According to this theory, at the beginning, all the mass-energy of the current Cosmos was within an extremely small, dense and hot singularity and after a kind of explosion, an expansion began and it actually keeps expanding up until today. In Hoyle's theory, density remains constant, whereas in the Big Bang theories, mass-energy remains constant.

There are three main setbacks in the model: The flatness problem, the horizon problem and the monopole problem.

To overcome the adversities presented by the Big Bang hypothesis, Alan Guth proposed the theory "of the inflationary model", according to which the universe suffered an exponentially accelerated expansion called "the inflation". Inflation is considered to have lasted between $10^{-36}$ and $10^{-35} \mathrm{sec}$.

Meanwhile, the Radius of the Universe grew from just over Planck's length to approximately one metre (Guth, 1999). 
Guth, in the appendix of his aforementioned publication, also contributed the idea that:

$$
\begin{aligned}
& \text { «energy is released when a gravitational field } \\
& \text { is created... which should be negative». }
\end{aligned}
$$

By 1973 some ideas have already been presented that defended that the entire Universe could arise from a fluctuation of the vacuum without violating the conservation laws, by compensating the positive energy of the mass with the negative gravitational energy (Fomin, 1973) and (Tryon, 1973).

Alexander Vilenkin contributed the theory that the universe was created out of nothing (Vilenkin, 1982).

Several authors do not believe in the Big Bang theory as a cosmological model and have proposed alternative theories. Peter Lynds proposed the model of a universe that is repeated an infinite number of times and as a consequence, time is cyclical (Lynds, 2006).

We should note that none of the theories that have been mentioned, including the Big Bang theory, relies on a mathematical-physical basis; these are speculative theories that have been more or less credible in their time. The Big Bang theory with the Inflation is currently accepted by the academic world.

Steven Weinberg in "The first three minutes" shows us how the primitive universe developed in preparation to evolve into the present cosmos (Weinberg, 1978).

However, the great question about the origin of the "initial singularity", the "primeval atom" or the "cosmic egg" remains. Krauss (2013) and other authors have proposed the idea that:

\section{«In quantum gravity, universes can and indeed always will, spontaneously appear from nothing. Such universes need not be empty, but can have matter and radiation in them, as long as the total energy, including the negative energy associated with gravity, is zero».}

Here we present a new equation that responds to our concerns, providing the new theory of the Cosmos with a mathematical-physical basis and explaining the origin of mass-energy in the Cosmos, with gravity playing a fundamental role. Moreover, this equation allows us to state that the total energy of the Cosmos is zero.

\section{Dimensional Expression of Mass}

Based on the following two basic expressions, $F=G \frac{M m}{D^{2}}$ expressed dimensionally as:

$$
[F]=\left[G M^{2} L^{-2}\right]
$$

$$
F=m a \text { expressed dimensionally as: }
$$

$$
[F]=\left[M L T^{-2}\right]
$$

Equating both expressions:

$$
\left[G M^{2} L^{-2}\right]=\left[M L T^{-2}\right]
$$

By simplifying the Mass term, we obtain:

$[M]=\left[G^{-1} L^{3} T^{-2}\right]$

To transform this expression into an equivalent expression with an easier interpretation, we can express the length " $L$ " as a function of the speed of light in the vacuum " $c$ " and the time " $T$ " in the following form:

$$
[L]=[c T]
$$

Obtaining the following expression:

$[M]=\left[c^{3} G^{-1} T\right]$

The constants $[G]=\left[L^{3} M^{-1} T^{-2}\right]$ and $[c]=\left[L T^{-1}\right]$ can be considered in their double function, either as a kind of dimensional parenthesis within the expression, or as universal constants, as long as the dimensional homogeneity of the expression is preserved.

This is a very simple deductive process that provides us with an extremely simple expression with a profound meaning.

In this expression $[M]=\left[c^{3} G^{-1} T\right]$, the mass " $M "$ is dimensionally a function of two universal constants " $c$ " and "G" (dimensional constants with values that depend on the system of units used) and a single variable of time " $T$ ".

Expression (4) is not a physical formula because it has been deduced from dimensional equations, which can mask the existence of non-dimensional constants. It can be converted into a physical formula by introducing a dimensionless constant " $K$ " in the following way:

$$
M=K c^{3} G^{-1} T
$$

Although expression 4 clearly shows that the mass " $M$ " grows proportionally with time, it is necessary to determine the value of the possibly dimensionless constant " $K$ ", which when added to the two dimensional constants " $c$ " and " $G$ ", transforms expression 4 into a physical equation that allows quantitative results to be obtained.

\section{Obtaining the Value of " $K$ " for Planck Time}

Directly using the expressions corresponding to the Planck Mass " $M_{P}$ " and Planck Time " $T_{P}$ " (Sanchez, 1997), we obtain the following: 


$$
\begin{aligned}
& M_{p}=\sqrt{\hbar c / G} ; T_{p}=\sqrt{\hbar G / c^{5}} ; M_{p} / T_{p}=c^{3} / G \\
& M p=c^{3} G^{-1} T_{p}
\end{aligned}
$$

For the particular case of the Planck Time, we verify that the dimensional expression of mass (4) corresponds with formula 5, which was obtained from the definition of the Planck units, i.e., the value of $K$ is 1 .

The correspondence between (4) and (5) shows that expression:

$$
M p=c^{3} G^{-1} T_{p}
$$

represents the correct physical equation.

\section{Interpreting the Equation $M_{p}=c^{\mathbf{3}} G^{-1} T_{p}$}

The interpretation of Eq. 6 provides us with a very different conception of the Cosmos from the established one:

1. For $T=0$, the mass $M=0$.

2. The mass grows proportionally with time. Therefore, we have the history of the mass of the Cosmos.

3. The relationship between the universal constants "c $3 / \mathrm{G}$ " seems to be generating the mass.

4. In Eq. 6, time "T" appears as an absolute value. However, it doesn't necessarily have to be the independent absolute variable. In expression 3 , if we substitute [T] for [L/c], instead of substituting [L] for [cT], we will obtain the following expression: $\mathrm{M}=\mathrm{c}^{2} \mathrm{G}^{-1} \mathrm{~L}$, rather than Eq. 6. In this case, "space" would be the absolute variable, " $L$ " the size of the radius of the Cosmos and thus, time " $T$ " would be a consequence, that should be interpreted as the magnitude that measures how light travels across the Cosmos. Taking all of this into consideration, for simplicity's sake, we will consider time " $T$ " as an absolute variable, from now on.

\section{Determination of the Growth Rate of the Mass of the Cosmos}

We can apply equation 6, to the history of the Cosmos. Assuming that the mass of the cosmos grows proportionally with time, we can calculate the mass of the Cosmos after the first second of existence and its growth rate per second, $\Delta M s^{-1}$, based on the following:

$$
G=6.674 \times 10^{-11} \mathrm{~m}^{3} \mathrm{~kg}^{-1} \mathrm{~s}^{-2} ; \mathrm{c}=3 \times 10^{8} \mathrm{~ms}^{-1}
$$

By derivation of the equation 6 we obtain $d M / d T=$ $c^{3} G^{-1} d M / d T=4 \times 10^{35} k g s ~^{-1} ; \Delta M s^{-1}=4 \times 10^{35} k g s^{-1}$.

Assume that the mass of the Sun is $2 \times 10^{30} \mathrm{~kg}$ (Martínez et al., 2005), which corresponds to $\Delta \mathrm{Ms}^{-1}=$ $2 \times 10^{5}$ suns per second.
That is, a mass equivalent to 200000 suns per second is generated.

\section{Determination of the Mass of the Cosmos Created from the Beginning}

The current cosmology estimates the present age as: 13.7 billion years, (WMAP, 2012). Since the beginning of the Cosmos, the elapsed time in seconds is approximately $4.32 \times 10^{17} \mathrm{~s}$

Applying the obtained growth rate, it gives us a total mass of the Cosmos today that is equivalent to $8.64 \times 10^{22}$ suns. According to our calculations $8.64 \times 10^{22}$ suns $\mathrm{x}$ $2 \times 10^{30} \mathrm{~kg}$ per sun $=1.73 \times 10^{53} \mathrm{~kg}$.

\section{Is this Mass Compatible with other Estimates}

In a simple, straightforward and unpretentious way: Assuming $10^{11}$ to $4 \times 10^{11}$ stars in our Galaxy (Masetti, 2015) and assuming $2.46 \times 10^{11}$ galaxies in the Cosmos (Conselice, 2016), we would have the equivalent of $2.46 \times 10^{22}$ to $10^{23}$ stars in the Universe.

Which is of the same order of magnitude calculated by the expression 6 of $8.64 \times 10^{22}$ suns.

The current estimate of the mass of the observable universe, obtained by other means, is $10^{53} \mathrm{~kg}$ (Deshpande and Yoshida, 2019), not far from of the one of our calculations $1.73 \times 10^{53} \mathrm{~kg}$.

\section{The Cosmos in Planck Time}

Using the formula for mass in expression 6 , we calculate the amount of mass that could be created in the so-called Planck Time. More decimals are used in order to achieve a higher precision:

$$
\begin{aligned}
& c=299792458 \mathrm{~ms}^{-1} ; \mathrm{G}=6.67408 \times 10^{-11} \mathrm{~m}^{3} \mathrm{~kg}^{-1} \mathrm{~s}^{-2} \\
& \text { In the Planck time, } T_{P}=5.39106 \times 10^{-44} \mathrm{~s} \\
& M_{P}=c^{3} G^{-1} T_{P} ; M_{P}=21.7643 \times 10^{-9} \mathrm{~kg}
\end{aligned}
$$

That is, 21.7643 micrograms, which coincides with the Planck Mass.

We can say that the Planck Mass " $M_{P}$ " was the mass of the Cosmos when its age was the Planck Time " $T_{P}$ ".

Given that mass is proportional to time, this allows us to affirm that the growth rate of the mass of the Cosmos is one Planck Mass for each unit of Planck Time elapsed.

The foregoing confirms that the deduced equation represents the evolution of the mass-energy of the Cosmos from Planck's time to the present and also in the future. 


\section{The Universal Constant "@"}

Equation 6 quantified the growth of the mass of the Cosmos and its value at any point in the evolution of the Cosmos. However, the reason for this creation of matter is not clear. In exchange for what is this mass being created? To support this understanding, we have introduced a new universal constant.

We consider that there are four interactions in nature and that at the beginning, they were all connected.

Close to the Planck Time, that is, at approximately $10^{-43}$ seconds after the start of everything, the first of these interactions, the Force of Gravity, became independent.

At approximately $10^{-37}$ seconds, the Strong Nuclear Force became independent.

Finally, at $10^{-12}$ seconds, the Weak Nuclear Force was separated from the Electromagnetic Force.

In this way, it turns out that the electromagnetic force, which is responsible for light, is a consequence of the last separation between interactions.

If we admit that the velocity of propagation of the gravitational fields, to which we assign the symbol "@", is identical to the speed of light in a vacuum "c" (e.g., Abbott B. P. 2017) and assuming that in the cosmic origin, the gravitational fields pre-existed the electromagnetic fields, we must admit that "@" is the cause and "c" is a consequence, so the propagation velocity of the gravitational fields "@" must be considered to be the true universal constant in substitution of "c".

Although "c" and "@" have quantitatively identical values, they are very different conceptually and we advise that the new constant should be introduced in deductions and calculations.

These ideas were explained by Pavía (2004) who argued that the appropriate equation for energy should be:

$$
E=m @^{2}
$$

\section{The Equation of the Mass-Energy of the Cosmos}

If in Eq. 6, we substitute "c" for "@", we obtain the final form of the equation sought, that is the equation of the mass-energy of the Cosmos:

$$
M=@^{3} G^{-1} T
$$

The "M", which represents mass-energy expressed in mass, is a function of two universal constants (in this case, both constants are related to gravitational fields) and a single variable, the time "T".

In this expression 8 , it is evident that the creation of mass-energy is the counterpart of the expansion of the Gravitational Field with $@^{3} G^{-1 "}$ being the part of the expression responsible for the creation of mass-energy, from Planck Time to the present and will continue to be so in the future. It also suggests that the formation of mass-energy occurs as a by-product of the continuous increase of the gravitational field, which generates space.

Considering expression 7 , the equation equivalent to 8 expressed in energy is:

$E=@^{5} G^{-1} T$

\section{The Substitution of "c" by "@"}

We have seen how the substitution of "c" by "@" provides a better interpretation of expression 6 when obtaining Eq. 8.

We must also remember that close to the Planck Time, the electromagnetic interaction was not decoupled, so there was no " $c$ ", which strengthens our proposal to generalize the substitution of " $c$ " with "@".

Therefore, we should express the Planck units as:

$$
M_{P}=\sqrt{\hbar @ / G} ; \quad T_{P}=\sqrt{\hbar G / @^{5}} ; \quad L_{P}=\sqrt{\hbar G / @^{3}}
$$

From them, we directly obtain the following:

$$
M_{P}=@^{3} G^{-1} T_{P}
$$

Likewise, the Planck Energy is given by:

$$
E_{P}=@^{5} G^{-1} T_{P}
$$

\section{Conservation of Energy and $M=@^{\mathbf{3}} \mathbf{G}^{-1} \mathbf{T}$}

The principle of energy conservation requires a first adaptation as a result of Einstein's formulation of the equation of energy as a function of the rest mass, $E=$ $m c^{2}$ (Stachel, 2001); from this first moment, the binomial "mass-energy" must be considered as the magnitude that remains constant.

The equation $M=@^{3} G^{-1} T$, where " $M$ " represents the continuous increase of mass-energy, forces us to reconsider for a second time the law of energy conservation. Now, we find ourselves not with a conservative system, but with an evolutionary system, in which the mass-energy, that we now express separately, increases without end:

$$
E / @^{2}+M=@^{3} G^{-1} T
$$

For the cited principle to conform to the conservative aspect, we must express it according to the following equation in a homogeneous way and referred to energy yields: 
$E+M @^{2}-@^{5} G^{-1} T=0$

The totality of the energy, mass and including the negative energy associated with gravity of the Cosmos remains equal to zero, regardless of the elapsed time.

\section{The Total Energy of the Cosmos is Zero}

In equation 10 the part of the energy associated with gravity " $G . E$ " it is:

$$
\text { G.E. }=-@^{5} G^{-1} T
$$

If we multiply and divide by "G@ T", we will have:

$$
\text { G.E. }=-G \text { @ }^{6} G^{-2} T^{2} / @ T ; G . E .=-G M^{2} / R
$$

This expression is not equivalent to the energy of gravitational cohesion or potential energy, since the latter represents the energy that has to be applied to the system so that it stops being united by the attraction of gravity, that is, separating the components infinitely. The value in this case of the Potential Energy, "P.E." would be P.E. = 3GM²/5R.

The following demonstrations are redundant, given the way in which we have obtained the energy associated with gravity. However, we consider them explanatory.

We present the formula $M=@^{3} G^{-1} T$, which shows that the mass-energy of the Cosmos grows proportionally with time. This expression is consistent with a view of the Cosmos as a gravitational sphere with a radius " $\mathrm{R}$ " that corresponds to the distance travelled at the propagation speed of the gravitational fields "@" in a time " $T$ ", that is $R=@ T$, wherein the mass and energy are given by expression $M=@{ }^{3} G^{-1} T$.

Energy of the rest mass is $E=@^{5} G^{-1} T$.

The energy associated with the gravity of the Cosmos applying $G . E=-G M^{2} / @ T$ yields $G . E .=-@{ }^{5} G^{-1} T$.

We observe that the energy associated with the gravity of the Cosmos has a negative sign and grows in absolute value with time. We can see that the two expressions are identical except for the opposite signs, i.e., the sum of the energy corresponding to the mass-energy created in a time " $\mathrm{T}$ " (as given by our formula) and the energy associated with gravity of the mass of the Cosmos with radius $\mathrm{R}=@ \mathrm{~T}$, is equal to zero.

We next re-formulate the problem in terms of Planck Time. We do not consider the formula for the mass of the Cosmos but instead Planck units are directly used as follows:

$M_{P}=\sqrt{\hbar @ / G} ; T_{P}=\sqrt{h G / @^{5}} ; L_{P}=\sqrt{\hbar G / @^{3}}$.

The energy equivalent to the Planck Mass is:

$E_{P}=M_{P} @^{2} ; \quad E_{P}=\sqrt{h @^{5} / G}$
The energy associated with gravity in Planck Time "G.E.TP" for a Planck Mass within a sphere with a radius of the Planck Length (that is, not within a cube) is:

$G . E_{\cdot T P}=-G M_{P}{ }^{2} / L_{P} ; G \cdot E_{\cdot T P}=-G \frac{\hbar @ / G}{\sqrt{\hbar G / @^{3}}} ; G \cdot E_{\cdot T P}=-\sqrt{h @^{5} / G}$

From the Planck units we obtain that the amount of the energy equivalent to the Planck Mass in Planck Time has the same absolute value but with the opposite sign as the energy associated with gravity, so that the resulting value of the of the sum is null.

According to Eq. 8, only one quantum leap was needed to generate our entire cosmos, from which gravity, with its gravitational constant " $G$ ", its propagation velocity "@" and time "T", arose.

It is from the Planck Time " $T_{P}$ ", with an initial mass equal to the Planck Mass " $M_{P}$ " and a space equal to a sphere of radius the Planck Length " $L_{P}$ ", that the Cosmos has been evolving, growing its radius and its mass-energy proportionally to time, while the energy associated to gravity has also grown with time the same value, but with a negative sign, being null the resulting value.

These results provide a new and clear vision of a Cosmos that was born out of nothing and, in energetic terms, is nothing.

\section{Discrepancies with the Steady-State Theory}

Given that mass is continuously being created, the cited formula 8 suggests that we are returning to the steady-state theory, as defended by Fred Hoyle, among others, in the middle of the 20th century. These cosmologists claimed that the Cosmos was invariant and that the decrease in its density caused by expansion, was compensated for by the continuous creation of mass.

Despite this apparent similarity to our proposal, there are two facts that completely differentiate our theory from the ideas of Hoyle and the followers of his theory:

- Our theory is consistent with an equation deduced mathematically from physical formulas. Whereas Hoyle and his followers defended their theory solely based on the idea of keeping the density of the Cosmos constant

- According to Hoyle, the created matter compensates for the decrease in density as a consequence of expansion. According to our formula, mass grows proportionally with time, while volume grows as the cube of time. Therefore, the density of the Cosmos decreases as a quadratic function of time, not fulfilling the requirements of the steady-state theory 


\section{Differents Methods to Obtain $M={ }^{3} \mathbf{G}^{-1} \mathbf{T}$}

At the beginning of this study we have obtained the equation $M=@^{3} G^{-1} T$, but in reality we have been able to achieve this expression in five different ways, which shows that it is hidden within many laws of physics and nature.

\section{Newton's equations}

The first one has been obtained from the two Newton's equations, using the Planck Units in order to get the dimensionless constant " $\mathrm{K}$ ".

\section{Planck Units}

The second one is a consequence of the expression obtained from the Planck Units, $M_{P}=@{ }^{3} G^{-1} T_{P}$, which is used to demonstrate that the value of " $\mathrm{K}$ " is the unit. If we multiply both sides in such equation by a positive integer " $N$ ", we obtain the following formula:

$$
M=N M_{P}=@^{3} G^{-1} N T_{P}
$$

Which clearly states that the mass grows proportionally with the elapsed "quanta of time" or "Plank times".

\section{Gravitational Interaction}

The third, considering that the first interaction to be decoupled was Gravity, with its universal constant "G" and its propagation velocity constant "@", at the same time that time "T" emerged, we resort to a procedure similar to the one used by Planck to obtain the system of "Planck Units". In this case we will consider that the fundamental dimensions can be expressed in terms of the two universal constants, "G" and "@" and of a variable, the time " $T$ ".

We try to obtain these dimensions (which we will call universal), universal Length " $L_{u}$ ", universal Mass " $M_{u}$ " and universal Time " $T_{u}$ " as a function of @, $G, T$.

For this we will use the powerful Dimensional Analysis.

The dimensional expressions of these constants and time are the following:

$$
[@]=\left[L T^{-1}\right] ; \quad[G]=\left[L^{3} M^{-1} T^{-2}\right] ; \quad[T]=[T]
$$

1. To express the universal Length $L u$, in terms of the two constants of Gravity and Time, we propose the following equation:

$$
L_{u}=@^{\alpha} G^{\beta} T^{\gamma}
$$

Matching the exponents:

$$
\begin{array}{ll}
(L) & 1=\alpha+3 \beta+0 \\
(M) & 0=0-\beta+0 \\
(T) & 0=-\alpha-2 \beta+\gamma
\end{array}
$$

With these three equations:

$$
\alpha=1 ; \quad \beta=0 ; \quad \gamma=1
$$

Achieving what we will designate Universal length equivalent to the radius of the Universe $L_{u}=@ T$

2. To obtain the universal Mass $\mathbf{M}_{\mathrm{u}}$, in terms the two constants of Gravity and Time, we propose the following equation:

$$
M_{u}=@^{\alpha} G^{\beta} T^{\gamma}
$$

Matching the exponents:

$$
\begin{array}{ll}
(L) & 0=\alpha+3 \beta+0 \\
(M) & 1=0-\beta+0 \\
(T) & 0=-\alpha-2 \beta+\gamma
\end{array}
$$

With these three equations:

$$
\alpha=3 ; \quad \beta=-1 ; \quad \gamma=1
$$

which results in the following expression for the Universal Mass:

$$
M_{u}=@^{3} G^{-1} T
$$

Such expression, coincides with the one that we defined as Mass of the Cosmos:

3. In order to get the universal time $T_{u}$, in terms of the two constants of Gravity and time, we propose the following equation:

$$
T_{u}=@^{\alpha} G^{\beta} T^{\gamma}
$$

Matching the exponents:

$$
\begin{array}{ll}
\text { (L) } & 0=\alpha+3 \beta+0 \\
(M) & 0=0-\beta+0 \\
(T) & 1=-\alpha-2 \beta+\gamma
\end{array}
$$

With these three equations:

$$
\alpha=0 ; \beta=0 ; \gamma=1
$$

Achieving what we will designate Universal Time: $T_{u}=T$. 


\section{Energy Associated with Gravity}

The fourth, from the energy associated with the gravity of the Cosmos. Using the Planck units, expressed in terms of @:

$$
M_{p}=\sqrt{h @ / G} ; T_{p}=\sqrt{h G / @^{5}} ; L_{p}=\sqrt{h G / @^{3}}
$$

In the Planck Time: we could consider the Cosmos as a sphere with a radius of the Planck Length " $L_{P}$ ", equivalent to the Planck Time multiplied by the propagation velocity of gravity @: $L_{P}=@ T_{P}$.

The equivalent energy of the Planck Mass is:

$$
E_{P}=M_{P} @^{2} ; \quad E_{P}=\sqrt{h @^{5} / G} .
$$

Since the energy associated with gravity " $G . E$ " inside a sphere of radius " $R$ " and total mass " $M$ " is:

$G \cdot E .=-G M^{2} / R$ we obtain:

$G \cdot E_{\cdot T P}=-G M_{P}{ }^{2} / L_{P} ; G \cdot E_{\cdot T P}=-G \frac{\hbar @ / G}{\sqrt{h G / @^{3}}} ; G \cdot E_{\cdot T P}=-\sqrt{h @^{5} / G}$

The energy associated with gravity in the Planck Time, has the same value with opposite sign, as the equivalent energy of the Planck Mass. In consequence, the total energy of the Cosmos in the Planck time is null.

Nowadays: If the total energy of the system in the Planck time was null, why shouldn't it still be null nowadays? This hypothesis has been stated by several cosmologists, although it has not been proved yet.

And similarly, to what we have seen in the Planck time, it could be assumed that currently, the radius of the Cosmos sphere must be $R=@ T$ and the corresponding energy of the mass of the Cosmos should be identical to the energy associated with gravity of any mass with a negative sign, so that the total energy is null.

The equivalent energy " $E$ " of the mass-energy of the Cosmos " $M$ " is:

$$
E=M @^{2}
$$

The energy associated with gravity "G.E." of the Cosmos in order that the total energy is null, should be:

$$
\begin{aligned}
& G . E .=-E \\
& G . E .=-G M^{2} / R ; R=@ T ; G . E .=-G M^{2} / @ T \\
& -G M^{2} / @ T=-M @^{2} ; M=@^{3} G^{-1} T
\end{aligned}
$$

Concluding that the mass of the Cosmos in order to fulfill the condition that the total energy of the Cosmos is zero, it must be formulated by an expression identical to the one already obtained $M=@^{3} G^{-1} T$.

This clarifies that the mass-energy cannot be constant over time, as proposed by the Big Bang model, since gravitational fields are constantly expanding and consequently their energy associated with gravity varies with time as $\mathrm{R}$ varies.

\section{Energy Equation}

Finally, the fifth one can be obtained from the energy equation $E=h v$ :

$$
\begin{aligned}
& {[E]=\left[h T^{-1}\right]} \\
& {\left[M @^{2}\right]=\left[J \cdot S T^{-1}\right]\left(h=J \cdot \mathrm{i}=\text { Joules.second }=F \cdot L \cdot T=G M^{2} L^{-2} \cdot L \cdot T\right)} \\
& {\left[M @^{2}\right]=\left[G M^{2} L^{-2} L T \cdot T^{-1}\right]} \\
& {\left[@^{2}\right]=\left[G M L^{-1}\right]} \\
& {[M]=\left[@^{2} G^{-1} L\right] ; \quad[L]=[@ T]} \\
& {[M]=\left[@^{2} G^{-1} @ T\right]} \\
& {[M]=\left[@^{3} G^{-1} T\right] ; M=K @^{3} G^{-1} T}
\end{aligned}
$$

The dimensionless constant " $\mathrm{K}$ " equals the unit, as it was done in the first case.

Such diversity of procedures that lead to the deduction of the same expression confirms its legitimacy.

\section{Dr. Dimitar Valev Calculations}

When we had practically finished our work, we came across a surprise; we found an article by Dr. Dimitar Valev from the Bulgarian Academy of Sciences (Valev, 2012).

Dr. Valev by means of Dimensional Analysis resorted to a similar procedure to the are used by Planck to obtain his units, introducing the constants $\mathrm{c}, \mathrm{G}$ and $\mathrm{H}$. "H" being the Hubble constant, with which he replaces the reduced Planck Constant " $\hbar "$.

In this way he obtains the following equation, $M=$ $c^{3} / G H$, which allows him to calculate the mass of the present Cosmos.

We must consider that the Hubble Constant " $H$ " is inappropriately called "constant" since it varies with time. When expressed in homogeneous units it is simply the inverse of the time of the Cosmos, $H=1 / T$, therefore when the Cosmos had half the present time the value of $\mathrm{H}$ should be double. Then the equation $M=c^{3} / G H$ is identical to $M=@^{3} G^{-1} T$.

Dr. Valev similarly calculates the density of the present Cosmos and obtains that it is proportional to " $H^{2 "}$, that is inversely proportional to " $T^{2 "}$, a value that coincides with the one obtained by us and coherent with the fact that the mass grows proportionally to time while the volume grows proportionally to the cube of time. 
We have the impression that Dr. Valev, in this article, treats the Hubble Constant " $\mathrm{H}$ " as just another constant, by saying:

"According to recent cosmology, the Hubble constant " $H$ " slowly decreases with the age of the universe. However, there are indications that other constants, especially the gravitational and fine structure constants, also vary with time. Therefore, the Hubble constant might deserve to be treated on the same level as the other constants».

Professor Valev has about a dozen publications after the above mentioned in the field of Cosmology, works that were previously unknown to us. In them he continues treating, clarifying and progressing questions related to this subject. For the sake of brevity, we will only refer to two of them, (Valev, 2010; 2013).

\section{Conclusion}

Equation $\mathrm{M}=@{ }^{3} \mathrm{G}^{-1} \mathrm{~T}$ breaks all our schemes.

We stated that matter produces gravitational fields. We have now deduced that gravitational fields produce mass (matter). According to equation 8, the expansion of the Gravitational Field is a counterpart of the creation of mass. Formula 8 shows us how the immense Cosmos is created with a pattern equivalent to a Planck Mass for each unit of Planck Time, that is, the mass of 200000 suns per second.

Since the interaction of gravity anticipated the electromagnetic interaction, the propagation speed of gravitational fields "@" must be considered as the true universal constant in substitution of "c".

Our generation of our entire Cosmos only required a quantum leap, out of which gravity emerged with its constant “ $G$ " and its propagation speed "@”, and also the time " $T$ ".

The total energy of the Cosmos remains equal to zero regardless of the elapsed time.

The Planck volume should be considered for a sphere of radius $L_{P}$ and not for a cube of side $L_{P}$, as Planck considered and is typically used to obtain the Planck density.

The simplicity, structure and beauty of expression 8 confirm the correct substitution of "c" with "@".

The diversity of procedures that have led us to derive the expression $M=@{ }^{3} G^{-1} T$, shows us that it is an inherent part of nature and confirms its legitimacy. It is very significant that independently, Dr. Dimitar Valev and we have reached convergent and complementary conclusions, a condition that guarantees that our paths of reflection and work are correct.

We think that Eq. 8 and the constant "@" will open a new and interesting field of research and study related to that which is very large and to the very essence of matter.

\section{Acknowledgments}

We deeply appreciate José Antonio Morales Lladosa and Kevin Alabarta Játiva, for their dedication and good advice that have allowed successive improvements in the text, argumentation, style and presentation. They have ensured that the text has a clear common thread from the beginning to the end.

Likewise, we also thank María García Boronat and Vicente Puig Climent, for their work in translating this document into English and its various preparatory versions.

\section{Funding Information}

This publication is directly financed by the authors.

\section{Author's Contributions}

Both authors have jointly developed this study on the slate of the Agrupación Astronómica de la Safor.

\section{Ethics}

The authors will address any ethical issues that may arise after publication of this article.

\section{References}

Abbott, B. P. (2017). Gravitational waves and GammaRays from a binary neutron Star Merger: GW170817 and GRB 170817A. arXiv:1710.05834[astro-ph.HE]

Conselice, C. J., Wilkinson, A., Duncan, K., \& Mortlock, A. (2016). The evolution of galaxy number density at $\mathrm{z}<8$ and its implications. The Astrophysical Journal, 830(2), 83. doi.org/10.3847/0004-637X/830/2/83

Deshpande, A., \& Yoshida, R. (2019). The deepest recesses of the atom. Scientific American, 320(6), 32-39. https://dialnet.unirioja.es/servlet/articulo?codigo $=69$ 39565

Fomin, P. I. (1975). Gravitational instability of a vacuum and the cosmological problem. Akademiia Nauk Ukrains koi RSR Dopovidi Seriia Fiziko Matematichni ta Tekhnichni Nauki, 831-835. https://ui.adsabs.harvard.edu/abs/1975DoUkr......831 F/abstract

Fridman. (1922). Sobre la curvatura del espacio. Zeitschrift für Physik. 10,377-386. doi.org/10.1007/BF01332580

Gamow, G. (1970). My World Line: An Informal. Biography. Physics Today 24, 3, 51 (1971); doi.org/10.1063/1.3022626

Guth, A. H. (1999). El universo inflacionario: Ed. Debate Pensamiento. Books ISBN-10: 84-8306-178-3.

Hoyle, F. (1948). A new model for the expanding universe. Monthly Notices of the Royal Astronomical Society, 108(5), 372-382. doi.org/10.1093/mnras/108.5.372 
Hubble, E. (1929). A relation between distance and radial velocity among extra galactic nebulae. Procedings of the National Academy of Sciences of the U.S.A. doi.org/10.1073/pnas.15.3.168

Isaacson, W. (2008). Einstein. Su vida y su universo. Ed. Debate. Books. ISBN-10: 978-84-8306-788-8.

Krauss, L. M. (2013). A universe from nothing: Why there is something rather than nothing. Atria Books. ISBN-10: 1451624468.

Lemaître, G. (1927). A homogeneous universe of constant mass and increasing radius accounting for the radial speed of extra-galactic nebulae. In Annals of the Scientific Society of Brussels (Vol. 47, pp. 49-59). http://adsabs.harvard.edu/pdf/1927ASSB...47...49L

Lynds, P. (2006). On a finite universe with no beginning or end. arXiv preprint physics/0612053. https://arxiv.org/abs/physics/0612053

Martínez, V. J, Galadí-Enríquez, D., Soler, E. M, \& Miralles, J. A (2005). Fundamental Astronomy (Vol. 81). University of Valencia. ISBN-10: 8437061040.

Masetti, M. (2015). How Many Stars in the Milky Way. https://asd.gsfc.nasa.gov/blueshift/index.php/2015/0 7/22/how-many-stars-in-the-milky-way/.

Pavía, F. (2004). mc ${ }^{2}$ versus m @ ${ }^{2}$, La constante universal "c" en cuestión. HUYGENS No 49 , https://www.astrosafor.net/Huygens/2004/49/Cosmolo gia.htm
Sanchez, D. R. (1997). Física cuántica. Ed. Pirámide Madrid. Books. ISBN-10: 84-368-1656-0.

Stachel, J. (2001). Einstein 1905: Un año milagroso. Editorial Crítica, S.L. Books. ISBN-10: 84-8432215-7.

Tryon, E. P. (1973). Is the universe a vacuum fluctuation?. Nature, 246(5433), 396-397. doi.org/10.1038/246396a0

Valev, D. (2010). Consequences from conservation of the total density of the universe during the expansion. arXiv preprint arXiv:1008.0933. https://arxiv.org/abs/1008.0933

Valev, D. (2012). Estimations of total mass and density of the observable universe by dimensional analysis. Aerospace Res. Bulgaria, 24, 67-76.

Valev, D. (2013). Three fundamental masses derived by dimensional analysis. Am. J. Space Sci, 1(2), 145-149. doi.org/10.3844/ajssp.2013.145.149

Vilenkin, A. (1982). Creation of universes from nothing. Physics Letters B, 117(1-2), 25-28. doi.org/10.1016/0370-2693(82)90866-8

Weinberg, S. (1978). The first three minutes of the universe; a modern conception of the origin of the universe (No. 04; QB981, W4.) Books ISBN 10: 0006348998

WMAP. (2012). Team receives the Gruber Cosmology Prize, data and results. https://wmap.gsfc.nasa.gov/news/ 